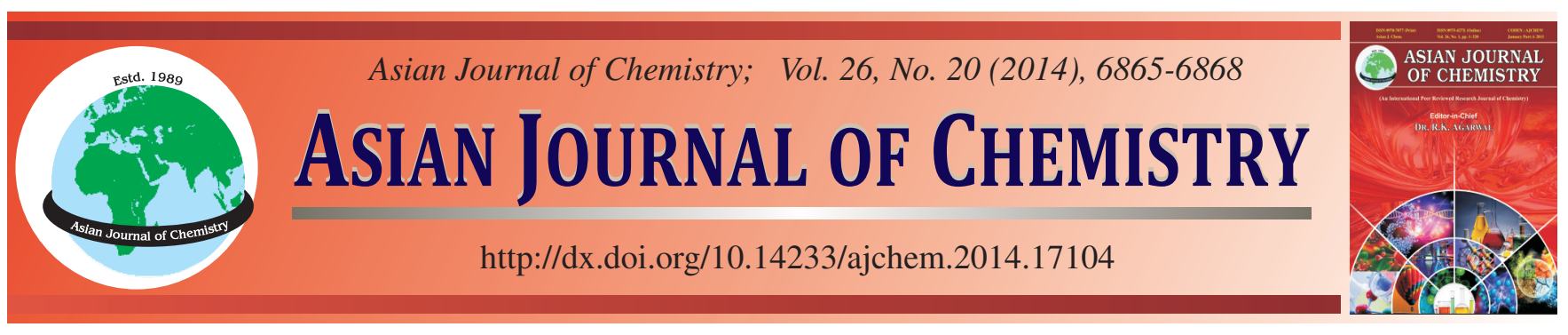

\title{
Simultaneous Analysis of Four Bioactive Compounds in Migan Granules Using Internal Addition Standard Method in Gas Chromatography
}

\author{
Guofang Xu ${ }^{1}$, Xiaohua $\mathrm{Li}^{1}$, Ping Liu ${ }^{1}$, Guanghui $\mathrm{Li}^{1}$, Zhihui Hua ${ }^{1}$ and Xiaosu $\mathrm{Li}^{2, *}$
}

${ }^{1}$ Zhengzhou People's Hospital, Zhengzhou 450003, Henan Province, P.R. China

${ }^{2}$ Henan Provincial People's Hospital, Zhengzhou 450000, Henan Province, P.R. China

*Corresponding author: Tel: +86 15038272612; E-mail: xiaosulicn@163.com

\begin{abstract}
A simple and reliable gas chromatography method with flame ionization detector was developed for the separation and quantification of four bioactive essential compounds including deoxyschizandrin, schizandrin B, schisandrol A and imperatorin in a traditional Chinese medicine preparation (TCMP), Migan granules. The analytes were distilled with steam distillation. The separation was achieved on an HP-5 phenyl methyl siloxane capillary column $(30 \mathrm{~m} \times 0.32 \mathrm{~mm}$, i.d. $\times 0.50 \mu \mathrm{m})$ with temperature programmed. The LODs and LOQs of the four analytes were less than 2.10 and $6.93 \mu \mathrm{g} / \mathrm{mL}$, respectively. The intra-day and inter-day coefficients of variation were less than 4.3 $\%$ (RSD) and the recoveries were in the range of 91.4-99.3\% for all four analytes. The validated method was successfully applied to simultaneously determine the four essential compounds in Migan granules.
\end{abstract}

Keywords: Gas chromatography, Traditional Chinese medicine, Essential oils, Internal addition standard method, Migan granules. |

\section{INTRODUCTION}

Migan granules, as a traditional Chinese medicine preparation (TCMP), is mainly used for the acute and chronic urinary system infection in clinic. The preparation comprises the extracts of nine medicinal herbs, including Bupleurum chinense DC., Schisandra chinensis (Turcz.) Baill., Polygonum aviculare L., Phellodendron chinense Schneid., Angelica dahurica (Fisch. ex Hoffm.) Benth. et Hook. f., Dispsacus asperoides C.Y. Cheng et T.M.Ai, Taxillus chinensis (DC.) Danser., Glycyrrhiza uralensis Fisch., Malva verticillata $\mathrm{L}^{1}$.

The essential oils from Schisandra chinensis (Turcz.) Baill (deoxyschizandrin, schizandrin B, schisandrol A) and Angelica dahurica (Fisch. ex Hoffm.) Benth. et Hook. f. (imperatorin) had antimicrobial, antifungal, antiviral and anti-inflammatory activity effect ${ }^{2-11}$. Therefore, their rapid and accurate quantification is of great significance in the quality control of this preparation. In the present work, our aim was to develop an accurate, specific and reproducible GC method for the simultaneous determination of deoxyschizandrin, schizandrin B, schisandrol A and imperatorin (Fig. 1) in Migan granules.

As the chemical compositions in traditional Chinese medicine are complex, various problems may be encountered even using the chromato-graphic method with the highest separation efficiency, such as the minute sample size, the large introduction error and the low precision of repeated injection in gas chromatography. These always result in failure in external standard method instrumental analysis. However, it is difficult to find a suitable internal standard substance for internal standard method because of the complicated chemical compositions in traditional Chinese medicine preparation. So, we considered another quantitative method, namely internal addition standard method (IAS). The method was adopted by spiking pure compound (standard) to the sample solution as the standard solution. Then the content of the analyte in sample solution can be calculated according to the increment of peak areas between the sample and standard solution. The delineation of the addition standard method is shown in Fig. 2. and the quantity formula can be shown in Formula 1.

$$
\mathrm{m}_{\mathrm{i}}=\Delta \mathrm{m}_{\mathrm{i}} \times \frac{\mathrm{A}_{\mathrm{i}}}{\Delta \mathrm{A}_{\mathrm{i}}}
$$

(Formula 1. The basic formula of internal addition standard method.)

In order to overcome the error of injection, a stable peak that has a similar retention time and peak area with the peak of analyte was chosen to be a reference peak (equivalent to internal standard peak) in the chromatogram (Fig. 2). The quantity of analyte is as the following formula indicated (Formula 2):

$$
\mathrm{m}_{\mathrm{i}}=\Delta \mathrm{m}_{\mathrm{i}} \times \frac{\mathrm{A}_{\mathrm{i}} / \mathrm{A}_{\mathrm{r}}}{\mathrm{A}_{\mathrm{i}}^{\prime} / \mathrm{A}_{\mathrm{r}}^{\prime}-\mathrm{A}_{\mathrm{i}} / \mathrm{A}_{\mathrm{r}}}
$$


<smiles>COc1cc2c(c(OC)c1OC)-c1c(cc(OC)c(OC)c1OC)CC(C)C(C)C2</smiles>

Deoxyschizan

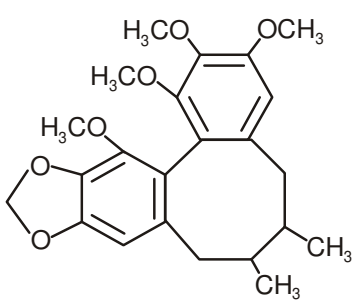

Schizandrin B

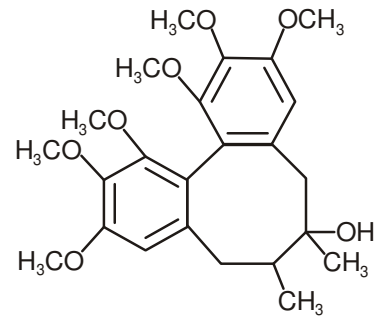

Schisandrol A

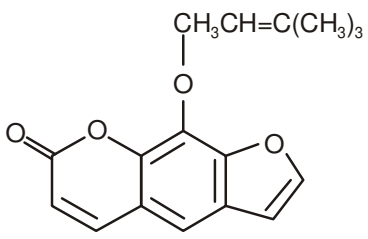

Imperatorin

Fig. 1. Chemial structures of Deoxyschizan, Schizandrin B, Schisandrol A, Imperatorin
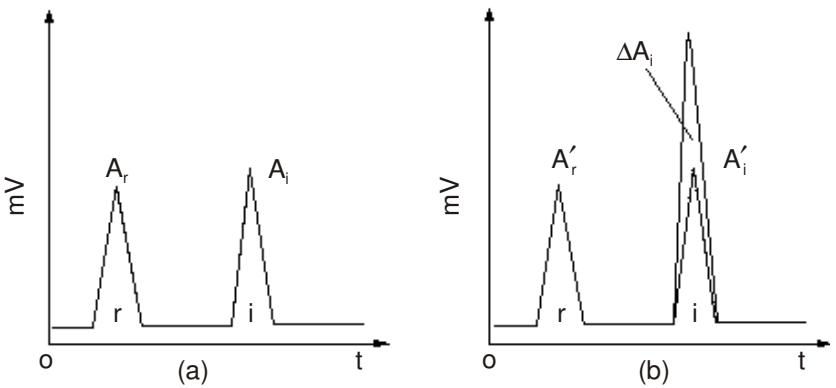

Fig. 2. Delineation of the addition standard method, (a) Chromatogram of the sample solution; (b) The chromatogram of standard solution. Peak $r$ was the reference peak; peak i the peak of analyte; $\Delta \mathrm{A}_{\mathrm{i}}$ was the increment of peak areas between the sample and standard solution

(Formula 2. The formula of internal addition standard method.)

In the formula, $\Delta \mathrm{m}_{\mathrm{i}}$ is the quantity of standard added into the sample solution; $A_{i}$ is the peak area of the analyte in the sample solution; $\mathrm{A}_{\mathrm{i}}$ ' is the total peak area of the analyte in the standard solution; $A_{r}$ is the peak area of the reference peak in the sample solution and $\mathrm{A}_{\mathrm{r}}{ }^{\prime}$ is the peak area of the reference peak in the standard solution.

In this study, an internal addition standard method for the simultaneous determination of four bioactive constituents in Migan granules was established. The method was precise and accurate and could be used for simultaneously determining the four bioactive compounds in Migan granules.

\section{EXPERIMENTAL}

Migan granules (Lot No.:070101, 070102, 070103) were gifted by Liaoning Yicheng pharmaceutical Co., Ltd. (Shenyang, China). The standards of deoxyschizandrin, schizandrin B, schisandrol A and imperatorin were obtained from the National Institute for the Control of Pharmaceutical and Biological Products (Beijing, China). The purity of these authentic compounds were determined to be more than $98 \%$ by normalization of the peak areas detected by gas chromatography and showed stable in $n$-hexane solution. $n$-Hexane was purchased from Concord Technology Co., Ltd (Tianjin, China).

Isolation of essential oils: The separated granules accurately weighed (12 g) was added to a round-bottomed flask containing $120 \mathrm{~mL}$ of water and was steam-distilled in a full glass essential oil determination apparatus as recommended by Chinese Pharmacopeia. The distillation was carried out for $3.5 \mathrm{~h}$ at $100{ }^{\circ} \mathrm{C}$. After the condensed material cooling down, the water and essential oils (about $5 \mathrm{~mL}$ ) were decanted into a $25 \mathrm{~mL}$ separatory funnel. After liquid-liquid extraction $(5+5$ $\mathrm{mL}$ ), the essential oils solution were dried over anhydrous sodium sulfate and diluted to $25 \mathrm{~mL}$ with $n$-hexane and then stored in dark at $4{ }^{\circ} \mathrm{C}$ prior to $\mathrm{GC}$ analyses.

Preparation of sample solutions and standard solutions: The standard stock solutions containing the four standards diluted with $n$-hexane to appropriate concentrations. The concentrations of deoxyschizandrin, schizandrin B, schisandrol $\mathrm{A}$ and imperatorin were $0.0600,0.240,0.300$ and $0.0600 \mathrm{mg}$ / $\mathrm{mL}$, respectively.

The essential oils solution of $1 \mathrm{~mL}$ was diluted to $10 \mathrm{~mL}$ with $n$-hexane as the sample solution, meanwhile another 1 $\mathrm{mL}$ of the essential oils solution and $1 \mathrm{~mL}$ of the standard stock solution were mixed and diluted to $10 \mathrm{~mL}$ with $n$-hexane as the standard solution.

Gas chromatography-analysis: Chromatographic separation and determination were performed on an Agilent 6890N Gas Chromatography system coupled with a flame ionization detector (FID) and a chemstation chromatography manager data acquisition and processing system. The extracted compounds were separated on an HP-5 phenyl methyl siloxane capillary column $(30 \mathrm{~m} \times 0.32 \mathrm{~mm}$, i.d. $\times 0.50 \mu \mathrm{m})$. The initial oven temperature was $150{ }^{\circ} \mathrm{C}$, held for $3 \mathrm{~min}$; ramped at 10 ${ }^{\circ} \mathrm{C} / \mathrm{min}$ to $210{ }^{\circ} \mathrm{C}$; a second rate at $5^{\circ} \mathrm{C} / \mathrm{min}$ to $240{ }^{\circ} \mathrm{C}$; a third rate at $10^{\circ} \mathrm{C} / \mathrm{min}$ to $280^{\circ} \mathrm{C}$, hold for $5 \mathrm{~min}$. Split injection was conducted with a split ratio of 1:50 and nitrogen of high-purity was employed as carrier gas with a constant flow of $1.2 \mathrm{~mL} /$ min. Hydrogen $(30 \mathrm{~mL} / \mathrm{min})$ and air $(300 \mathrm{~mL} / \mathrm{min})$ were used as fuel gases.The injector and detector temperatures were fixed at 230 and $280^{\circ} \mathrm{C}$, respectively. Representative chromatograms of the four standard compounds, the sample and standard solution were shown in Figs. 3-5.

Calibration curves and limits of detection and limit of quantification: Calibration curves were constructed by spiking the essential oils solution $(0.5 \mathrm{~mL})$ with known amounts of standard stock solutions $(0-3.5 \mathrm{~mL})$, then diluted to $10 \mathrm{~mL}$ with $n$-hexane. The values of pure samples (without standard added) were subtracted from the values obtained for samples spiked with standards. The least square method was used to calculate the regression equation. Five concentrations of the solutions were injected in triplicate and then the calibration curves were constructed by plotting the concentration of 


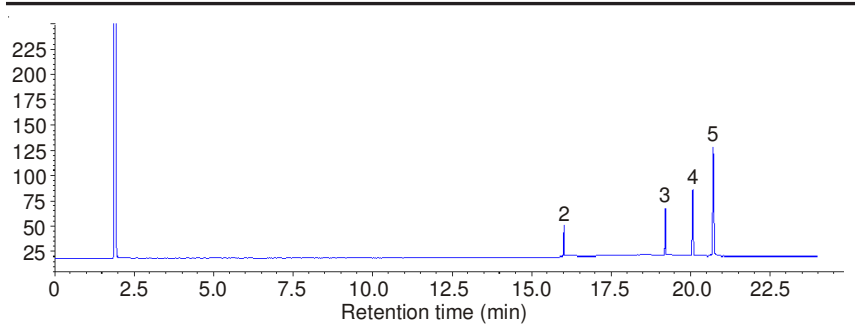

Fig. 3. Representative gas chromatogram of the four standard compounds in $n$-hexane. Peak: 2. Imperatorin; 3. deoxyschizandrin; 4. schizandrin B; 5. schisandrol A

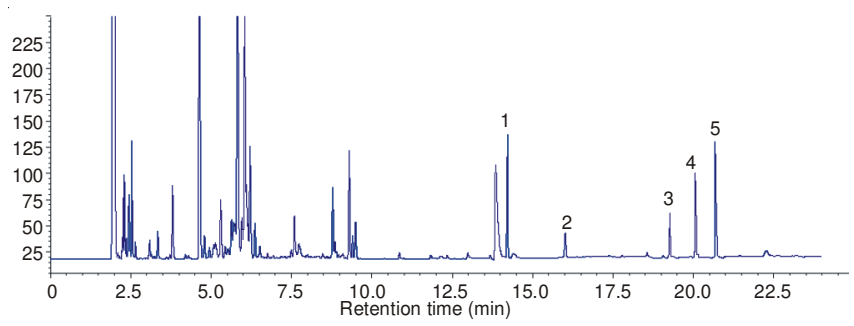

Fig. 4. Representative gas chromatogram of the sample solution. Peak: 1. reference; 2. imperatorin; 3. deoxyschizandrin; 4. schizandrin B; 5. schisandrol A

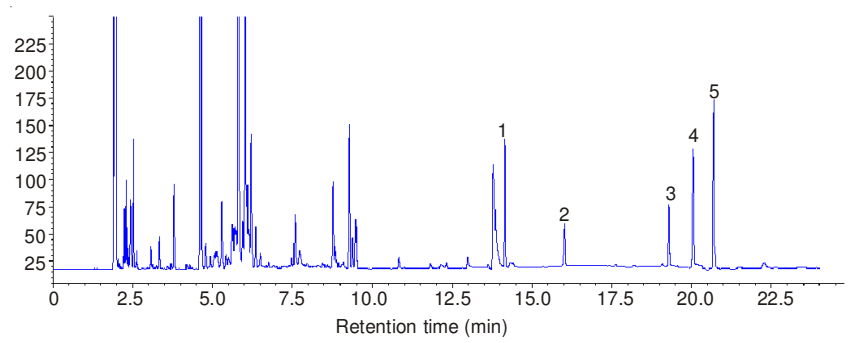

Fig. 5. Representative gas chromatogram of the standard solution. Peak: 1. reference; 2. imperatorin; 3. deoxyschizandrin; 4. schizandrin B; 5. schisandrol A

each analyte as a function of peak area ratio of analyte to reference.

The dilute solution of the four analytes was further diluted to a series of concentrations with $n$-hexane for the gain of the limits of detection (LOD) and limit of quantification (LOQ). The LOD and LOQ under the present chromatographic conditions were determined at a signal-to-noise $(\mathrm{S} / \mathrm{N})$ ratio of 3 and 10 , respectively. LOD and LOQ for each analyte were also shown in Table-1.

Precision and accuracy: The measurements of intra- and inter-day variability were utilized to assess the precision of the developed assay method. Migan granules of a batch were analyzed in six times within the same day to determine the intra-day precision, while the inter-day precision was determined by analyzing the same batch on four separate days. The relative standard deviation (R.S.D.) was taken as a measure of precision.
Recovery was used to evaluate the accuracy of the method. The recoveries for the four analytes of the method were tested on fortified samples at different concentrations. To determine the recovery, the contents of the four analytes in a sample were estimated previously. Different amount of standards was spiked into the sample at 0.8, 1.0, 1.2 times of the estimated mass of each analyte present in the sample, respectively, in triplicate. Then, the fortified samples were distilled and analyzed with the method, each in triplicate. The recovery for each standard compound was evaluated by comparing the difference of the compound mass between the fortified samples and the original sample with the nominal mass of the standard compound spiked in the fortified samples.

\section{RESULTS AND DISCUSSION}

Internal addition standard method: When the content of the analyte was calculated by internal addition standard method, the relative peak area was used to replace the absolute peak area. So we can overcome the instability factors such as the error of injection, the fluctuation of equipment, which may cause the quantitative error. The internal addition standard method is especially suitable to the samples with complicated components in which it's difficult to insert an internal standard peak.

In the method, the reference peak belongs to a compound which is unknown, but that doesn't affect analysis. Even, a different peak selected to be the reference peak doesn't affect analysis, too.

Isolation of essential oils: The influence of the volume of water and distillation time on the essential oils yield was studied. It can be seen that the water volume had no significant influence (Fig. 6). Therefore, $120 \mathrm{~mL}$ of water was used. The influence of distillation time on the distillation efficiency was shown in Fig. 7. Finally, the distillation was operated for $3.5 \mathrm{~h}$.

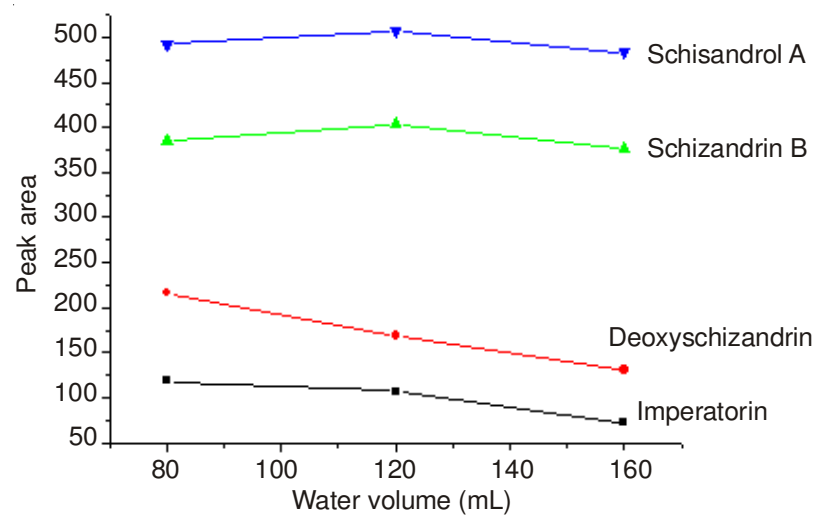

Fig. 6. Effect of the water volume on the extraction efficiencies of deoxyschizan, schizandrin B, schisandrol A and imperatorin in Migan granules

TABLE-1

LINEAR REGRESSION DATA, LOD AND LOQ OF INVESTIGATED COMPOUNDS FROM MIGAN GRANULES

\begin{tabular}{cccccc}
\hline Compounds & Calibration curve $^{\mathrm{a}}$ & $\mathrm{R}^{2}$ & Test range $(\mu \mathrm{g} / \mathrm{mL})$ & $\mathrm{LOD}(\mu \mathrm{g} / \mathrm{mL})$ & $\mathrm{LOQ}(\mu \mathrm{g} / \mathrm{mL})$ \\
\hline Deoxyschizandrin & $\mathrm{y}=9.35 \times 10^{-2} \mathrm{x}+6.40 \times 10^{-3}$ & 0.9989 & $3.39-24.4$ & 0.424 & 1.40 \\
Schizandrin B & $\mathrm{y}=5.64 \times 10^{-2} \mathrm{x}-3.69 \times 10^{-2}$ & 0.9977 & $12.9-96.9$ & 1.610 & 5.31 \\
Schisandrol A & $\mathrm{y}=4.39 \times 10^{-2} \mathrm{x}-4.59 \times 10^{-2}$ & 0.9995 & $16.8-122$ & 2.100 & 6.93 \\
Imperatorin & $\mathrm{y}=5.31 \times 10^{-2} \mathrm{x}-1.81 \times 10^{-2}$ & 0.9997 & $3.36-24.4$ & 0.420 & 1.39 \\
\hline
\end{tabular}

Note: ${ }^{a} \mathrm{y}$ : peak area ratio (peak area ${ }_{\text {spiked }}-$ peak area $\left.{ }_{\text {internal standard }}\right)$; $\mathrm{x}$ : concentration of compound $(\mu \mathrm{g} / \mathrm{mL})$ 


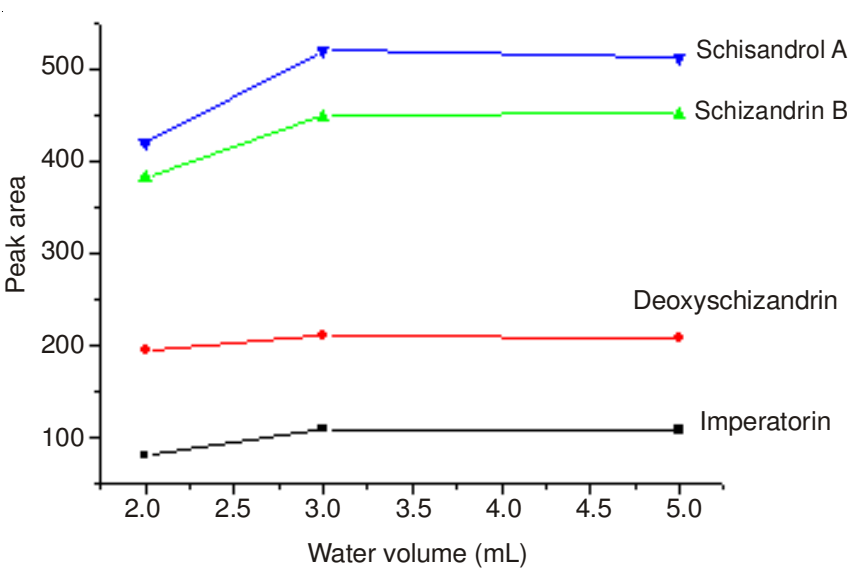

Fig. 7. Effect of extraction time on the extraction efficiencies of deoxyschizan, schizandrin B, schisandrol A and imperatorin in Migan granules

Calibration curves and limits of detection and quantification: After replicated analyses of calibration solutions, the calibration curves and correlation coefficients for the four analytes in $n$-hexane solution were obtained (Table-1). The correlation coefficients $\left(R^{2}\right)$ values are more than 0.997 , which show the method has good linearity. The limits of detection and limit of quantification for the four analytes are much lower than their concentrations in sample solutions. This shows that the proposed method is sensitive enough to analyze deoxyschizandrin, schizandrin B, schisandrol A and imperatorin in Migan granules.

Precision and accuracy: The relative standard deviation (R.S.D) were $2.3,1.8,2.9$ and $1.9 \%$ in intra-day assay and $3.8,2.5,4.3$ and $3 \%$ in inter-day assay for deoxyschizandrin, schizandrin B, schisandrol A and imperatorin, respectively. The mean recoveries were 96.7 (RSD 3.6), 99.3 (RSD 4.8), 93.5 (RSD 4.6) and $91.4 \%$ (RSD $4.0 \%$ ) for deoxyschizandrin, schizandrin B, schisandrol A and imperatorin, respectively.

Determination of four compounds in Migan granules: The four compounds in three batches of Migan granules were determined by the proposed gas chromatographic method at the conditions described above. Data of the quantitative analyses were expressed as mean \pm deviation (Table-2). The results showed that the contents of the four compounds were obvious differences among batches, so further researches might be carried on to define the limit of content for each analyte in Migan granules to control the quality of the preparation and ensure the efficacy of clinical application.
TABLE-2

CONTENTS OF DEOXYSCHIZANDRIN, SCHIZANDRIN B, SCHISANDROL A AND IMPERATORIN IN THREE BATCHES OF MIGAN GRANULES $(\mathrm{n}=3)$

\begin{tabular}{cccc}
\hline \multirow{3}{*}{ Components } & \multicolumn{3}{c}{ Content $(\mathrm{mg} / \mathrm{g})$} \\
\cline { 2 - 4 } & Lot no. & Lot no. & Lot no. \\
& 070101 & 070102 & 070103 \\
\hline Deoxyschizandrin & $0.106 \pm 0.008$ & $0.084 \pm 0.012$ & $0.128 \pm 0.015$ \\
Schizandrin B & $0.416 \pm 0.017$ & $0.402 \pm 0.009$ & $0.397 \pm 0.020$ \\
Schisandrol A & $0.602 \pm 0.035$ & $0.594 \pm 0.024$ & $0.628 \pm 0.019$ \\
Imperatorin & $0.133 \pm 0.012$ & $0.152 \pm 0.018$ & $0.167 \pm 0.023$ \\
\hline
\end{tabular}

\section{Conclusion}

In this paper, we developed a gas chromatography method for the simultaneous determination of four volatile constituents in Migan granules. The proposed method dissolved the problems of low precision in gas chromatography and difficulties of finding a good internal standard in complex samples analyses. In addition, this was the first report on the simultaneous quantification of the four analytes and the results demonstrated that the developed method could be applied as a reliable and sensitive quality control procedure for Migan granules.

\section{REFERENCES}

1. Chinese Ministry of Health, Standard for Chinese Patent Medicines, Part of Medical Renal System, No. WS-11401(ZD1401)-2002, Chemical Industry Press, Beijing, p. 26 (2003).

2. M. Zhu, K.F. Lin, R.Y. Yeung and R.C. Li, Ethnopharmacol., 67, 61 (1999).

3. P.Y. Chiu, M.H. Tang, D.H. Mak, M.K. Poon and K.M. Ko, Free Radic. Biol. Med., 35, 368 (2003).

4. S.P. Ip, M.K. Poon, C.T. Che, K.H. Ng, Y.C. Kong and K.M. Ko, Free Radic. Biol. Med., 21, 709 (1996).

5. P. Qiangrong, T. Wang, Q. Lu and X. Hu, Biochem. Biophys. Res. Commun., 335, 406 (2005).

6. Y. Wei and Y. Ito, J. Chromatogr. A, 1115, 112 (2006).

7. W. Ketai, L. Huitao, C. Xingguo, Z. Yunkun and H. Zhide, Talanta, 54, 753 (2001).

8. D. Lechner, M. Stavri, M. Oluwatuyi, R. Pereda-Miranda and S. Gibbons, Phytochemistry, 65, 331 (2004).

9. H. Sun, F. Wu, A. Zhang, W. Wei, Y. Han and X. Wang, J. Sep. Sci., 36, 485 (2013)

10. S. Zhu, Y. Wang, M. Chen, J. Jin, Y. Qiu, M. Huang and Z. Huang, Am. J. Chin. Med., 40, 551 (2012).

11. H. Wei, L. Sun, Z. Tai, S. Gao, W. Xu and W. Chen, Anal. Chim. Acta, 662, 97 (2010) 